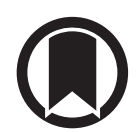

CrossMark

\title{
Tuberculosis among asylum seekers in Milan, Italy: epidemiological analysis and evaluation of interventions
}

\author{
Simone Villa', Luigi Ruffo Codecasa ${ }^{2}$, Marino Faccini ${ }^{3}$, Mirella Maria Pontello ${ }^{4}$, \\ Maurizio Ferrarese ${ }^{2}$, Paola Francesca Castellotti ${ }^{2}$, Sabrina Senatore ${ }^{3}$, \\ Anna Lamberti ${ }^{3}$, Ester Mazzola ${ }^{2}$, Carlo Federico Perno², Daniela Campisi², \\ Matteo Saporiti ${ }^{2}$ and Mario Carlo Raviglione ${ }^{5}$
}

Affiliations: ${ }^{1}$ Dept of Health Sciences, University of Milan, Milan, Italy. ${ }^{2}$ Regional TB Reference Centre, Villa Marelli Institute and Laboratory/ASST Niguarda, Milan, Italy. ${ }^{3}$ Health Protection Agency, Metropolitan Area of Milan, Milan, Italy. ${ }^{4}$ University of Milan, Milan, Italy. ${ }^{5}$ Global Health Centre, University of Milan, Milan, Italy.

Correspondence: Mario Carlo Raviglione, Global Health Centre, University of Milan, Via Festa del Perdono, 7, 20122, Milan, Italy. E-mail: mario.raviglionedunimi.it

@ERSpublications

Asylum seekers are vulnerable to TB owing to migration-related events and marginalisation in destination countries. Coordinated screening and surveillance programmes using well-informed datasets can reduce TB transmission, improve care and address LTBI. http://bit.ly/2SPp0uZ

Cite this article as: Villa S, Codecasa LR, Faccini M, et al. Tuberculosis among asylum seekers in Milan, Italy: epidemiological analysis and evaluation of interventions. Eur Respir J 2019; 54: 1900896 [https://doi. org/10.1183/13993003.00896-2019].

ABSTRACT In countries of the European Union, tuberculosis (TB) mainly affects marginalised people, including asylum seekers. Migratory flows from high-incidence countries to Italy have increased up to 2017, posing challenges to the national health system. This study sought to assess TB and latent TB infection (LTBI) prevalence among asylum seekers in Milan during the biennium 2016-2017 and to evaluate interventions in place.

A two-level active surveillance and screening system was developed for both TB and LTBI. Asylum seekers underwent an initial screening with a tuberculin skin test (TST) and a questionnaire at the receiving sites. At the Regional TB Reference Centre, those with a positive result underwent chest radiography. People aged $<35$ years with negative chest radiography results underwent further testing by interferon- $\gamma$ release assay. If results of the assay were positive, LTBI treatment was offered. TB and LTBI prevalence were compared with literature data.

A total of 5324 asylum seekers, mostly young (10-39 years; 98\%), male (84\%) and from sub-Saharan Africa (69\%), were enrolled in the study. 69 active TB cases were diagnosed and 863 LTBI-positive individuals were detected. TB prevalence was high (1236 per 100000 population) and LTBI prevalence was 28\%. Despite losses $(41 \%)$ during the transition from initial screening sites and the diagnostic centre, a good TB cure rate (84\%) and optimal LTBI treatment completion (94\%) were achieved.

Our study shows that TB incidence is high among asylum seekers in Milan and that well-coordinated screening measures are critical for early diagnosis and treatment. It also proves that rolling out successful at-scale interventions for both prophylaxis and disease management is feasible. 


\section{Introduction}

In low-incidence countries of the European Union (EU), vulnerable populations are largely responsible for the incidence of tuberculosis (TB) not declining at a faster rate [1]. Poor and marginalised people living in metropolitan areas have a greater risk of developing TB $[2,3]$. In the last few years, a large proportion of TB cases in the EU occurred among the foreign-born [4] at a younger median age than of native populations with TB $[1,5]$.

Among the foreign-born, particular attention must be paid to migrants owing to the increase in recent years in migratory flows from TB high-burden countries in sub-Saharan Africa and the Indian subcontinent [6,7]. This phenomenon, which also involves people seeking international protection, has led to further awareness of and increased concern about preventing infectious disease spread, particularly at entry points [8]. Asylum seekers may remain for months within centres awaiting an asylum application response, increasing the potential risk for both exposure and subsequent new infection and latent $\mathrm{TB}$ infection (LTBI) reactivation. Both in their original countries and during the migration process, asylum seekers are a particularly vulnerable population because of their exposure to various social and economic determinants $[9,10]$, such as poor and crowded living conditions, mal- or under-nutrition, poverty, stress, conflict and lack of education. In recipient countries, these determinants still represent a serious concern that is worsened by cultural barriers and healthcare access difficulties [11].

Although the risk of transmission to the native population is low, outbreaks may occur among migrants due to poor living conditions. For this reason, in many EU Member States different TB surveillance and screening systems, specifically built around asylum seekers, have been launched to detect active TB cases and prevent TB development by treating LTBI. In general, screening programmes consist of a combination of chest radiography, symptom-based assessment and, depending on the age and estimated TB incidence in the country of origin, LTBI detection with a tuberculin skin test (TST) and/or interferon- $\gamma$ release assay (IGRA) $[12,13]$.

Despite the efforts made by EU countries, the average treatment success rate for active TB has been reported as $60 \%-87 \%$ [14]. Important losses in screening completion have been observed for LTBI screening and management strategies [15], especially in the steps of treatment acceptance and initiation. Loss to follow-up has prevented higher preventive treatment completion rates, which are reported as $72 \%-80 \%$ for short regimens and $7 \%-83 \%$ for long regimens $[15,16]$. Furthermore, TB unawareness and stigma play major roles in delaying early diagnosis and compromising patient adherence to treatment [17].

Notably, in Italy 360000 migrants were hosted in reception centres during the 2016-2017 study period. Among all the Italian regions, Lombardy ranks first for the number of hosted asylum seekers (13\% of the national territory) and the Milan area accommodates $27 \%$ of asylum seekers present in the region. The time spent by asylum seekers in reception facilities depends on the time required by the Territorial Commissions to make decisions about asylum application. About $40 \%$ of requests received a positive response during the study period, on average 266 days after application. Our study aimed to assess the prevalence of both TB and LTBI among asylum seekers as soon as possible after their admission to reception centres in the city of Milan. We also investigated the risk of recent transmission within receiving sites. Finally, the performance of the city screening and treatment programmes during the biennium 2016-2017 were evaluated in terms of outcome of TB treatment and acceptance of, and adherence to, LTBI treatment.

\section{Methods}

The study focused on asylum seekers hosted within Milan's reception centres during the period January 1, 2016, to December 31, 2017. To tackle the TB health problem in asylum seekers arriving from high-incidence countries, a two-level active surveillance system has been developed in Lombardy to screen this population for both active disease, using a questionnaire (QS), and LTBI, using the TST [18].

\section{Data collection and record linkage}

There were four sources of data used: the Health Protection Agency of the Metropolitan Area of Milan; the Regional TB Reference Centre, Villa Marelli Institute, Milan; the regional database for infectious diseases; and the Microbiological and Virologic Laboratory, Niguarda Hospital, Milan. The Health Protection Agency data collection included information on screening activities (TST and QS) as well as personal information. The Villa Marelli Institute provided information on TB diagnosis, TB treatment, IGRA results and preventive therapy. The regional database was consulted to gather information on $\mathrm{TB}$ diagnosis and treatment. The Microbiological and Virologic Laboratory, which holds the regional strain collection, provided the molecular analysis of TB culture-positive samples. Data collection included follow-up information in 2018 from TB and LTBI cases diagnosed during the biennium 2016-2017. 
Data were first cleaned by removing duplicated records and transcription errors. To trace patients across the different databases, a stepwise deterministic strategy for record linkage was used. This approach began by examining the fields containing "name and surname", "country of origin", "date of birth", "hosting reception centre", "TST result" and "date of TST examination" from the Health Protection Agency database. Two records were considered to match if all or at least three identifiers were identical, provided that an identical correspondence between screening identifiers ("TST result" and "date of TST examination") in the analysed datasets was observed. If only two identifiers were matched, further verification was performed by reviewing the QS to ensure that the records identified by the electronic databases belonged to the same person. Two records were considered unmatched if the abovementioned criteria were not fully satisfied. Therefore, unmatched records were considered as losses between the first and second step of the system algorithm.

In case of one or more missing identifiers, record linkage was made possible by analysing data from medical records stored at Villa Marelli Institute confirming asylum seekers' identification ("identification documents check").

Analysing registration validity and internal completeness is crucial in order to understand the main problems in data collection. To analyse data registration validity, the database previously compiled by the Health Protection Agency was used to identify duplicated records (identical personal data) and, after data cleaning and record linkage, to find errors (e.g. typing "01/31/2000" for date of birth instead of “31/01/2000"). After data cleaning and record linkage, internal completeness was evaluated by comparing the number of completed data fields to the total number of data fields [19].

\section{Active TB surveillance, screening and treatment}

Asylum seekers hosted in receiving facilities could at any time self-report symptoms compatible with TB requiring further radiological and microbiological evaluations. Furthermore, whenever an asylum seeker with a previous negative screening test reported symptoms compatible with TB, as signalled to the Public Health Agency by reception centre staff, a chest radiograph was obtained.

The QS used to screen asylum seekers for active TB, developed with modifications from the one created by SchneEBERger GeIsLer et al. [20], contained 10 questions allowing scoring for a total of 22 points: 12 points for socio-demographic and history data ( 2 points if from a country with a TB incidence $>50$ per 100000 population; 1 point if arrived in Italy $<1$ year ago; 1 point if homeless; 4 points if previously treated for TB; 4 points in case of TB exposure from family and household members) and 10 for symptoms (2 points for each of cough for $\geqslant 3$ weeks, nights sweats, weight loss in the last 3 months, fever, chest pain). A threshold of 5 points was arbitrarily selected to consider the QS positive for suspected active TB.

Those with a positive result ( $Q S \geqslant 5$ points) were offered chest radiography and a medical examination at Villa Marelli Institute, the Regional TB Reference Centre of the City of Milan, or at a different facility in Milan (e.g. an emergency room) depending on the urgency of the symptoms. In all cases, experienced pulmonologists and radiologists assessed the presence of clinical and radiological signs suggestive of active TB. Individuals with positive chest radiography results underwent further microbiological evaluation (smear microscopy, nucleic acid amplification test and culture of biological samples). If the results of any such test were positive, or if a decision to start treatment was made on an empirical basis, the asylum seeker was offered anti-TB treatment. Classification of the case followed the EU case definitions of 1) "possible", with clinical criteria that included signs, symptoms or chest radiography suggestive of $\mathrm{TB}$, or with a clinician's decision to treat the person with a full course of anti-TB therapy; 2) "probable", if in addition to clinical criteria one of the microbiological criteria (positive sputum smear microscopy for acid-fast bacilli, nucleic acid amplification test or histological appearance of granuloma) was present; and 3) "confirmed", if confirmatory microbiological criteria (isolation of Mycobacterium tuberculosis from a clinical specimen or positivity for both nucleic acid amplification test and microscopy for acid-fast bacilli) were met [21]. The presence of anti-TB drug resistance was investigated in all strains of M. tuberculosis through a nucleic acid amplification test (Xpert ${ }^{\oplus}$ MTB/RIF; Cepheid, Sunnyvale, CA, USA) and phenotypic drug susceptibility testing (DST) for culture-positive samples [22]. Following TB diagnosis, a blood test was systematically performed to detect HIV infection. The treatment regimen was chosen according to DST results, immunological status and disease location (pulmonary versus extrapulmonary according to the World Health Organization (WHO) definition [23]).

The standard anti-TB regimen based on WHO guidelines consisted of 6-9 months of therapy with an intensive phase based on the combination of rifampicin, isoniazid, pyrazinamide and ethambutol (RHZE) for 2 months followed by 4 months of rifampicin and isoniazid with or without ethambutol (RH(E)), which is added in case of intolerance or resistance to pyrazinamide [24].

Treatment outcomes were defined according to WHO definitions [23]. 
Molecular surveillance, based on the 24-locus mycobacterial interspersed repetitive units/variable-number of tandem repeats (24-MIRU/VNTR) typing system, was also implemented to assess the likelihood of recent transmission. Clustering criteria for classifying recent TB transmission consisted of having an exact 24-MIRU/VNTR profile in at least two different patients' sputum samples or having 23 loci matches and one locus with one more or less repetition [25]. These criteria were assessed jointly with epidemiological data originating from contact tracing [26]. Recent transmission was considered by assessing strain characteristics comparing the 24-MIRU/VNTR profile, while reactivation cases were defined if a unique 24-MIRU/VNTR strain type was obtained.

\section{LTBI screening and preventive treatment}

Asylum seekers within reception facilities were additionally tested using TST, using the Mantoux method, to screen for LTBI. TST results were considered positive if the induration had a diameter of $\geqslant 10 \mathrm{~mm}$. Those presenting with a positive result were offered chest radiography and a medical examination at Villa Marelli Institute, where experienced pulmonologists and radiologists assessed the presence of clinical and radiological signs suggestive of active $\mathrm{TB}$ or the need for treatment of latent infection. Asylum seekers with a positive TST result, without radiological and clinical evidence of TB, underwent further testing with IGRA and for HIV infection if aged $<35$ years or in the presence of specific risk factors (e.g. recent TB contact). In children aged $<5$ years, preventive therapy was administered without additional tests. IGRA testing was performed using QuantiFERON-TB Gold In-Tube (QFT-GIT; QIAGEN, Hilden, Germany) from January 1 to December 13, 2016, and QuantiFERON-TB Gold Plus ${ }^{\circledR}$ (QFT-Plus; QIAGEN) from December 14, 2016. If the IGRA was positive, asylum seekers were offered preventive therapy for LTBI [27]. The proportion of acceptance of preventive therapy was defined as the number of those initiating treatment divided by the total number of individuals to whom it was offered (all IGRA-positive individuals and those aged $<35$ years who refused an IGRA even after a positive TST).

The LTBI preventive treatment regimens generally chosen by Villa Marelli Institute pneumologists consisted of a combination of rifampicin and isoniazid (Rifinah ${ }^{\oplus}$; Sanofi, Paris, France) for 3 months. Other single-drug regimens (rifampicin-only for 4 months or isoniazid-only for 9 months) were used in the presence of clinical risks such as high levels of liver enzymes, or in case of adverse drug reactions.

Treatment completion was defined as the proportion of asylum seekers who completed preventive therapy, assessed by patient self-reporting, divided by the number of those who started it.

\section{Statistical analysis}

Categorical variables were the number of asylum seekers and the corresponding percentage; continuous variables were summarised through the median and the quartiles (Q1-Q3). Missing and incomplete data were considered as "unknown" during statistical analysis.

TB prevalence rates were determined after performing age and sex standardisation for the total study population and for specific subsets, grouping individuals by WHO regions, geographical areas (United Nations regions) of origin and estimated TB prevalence in country of origin where available. The WHO regions-specific prevalence rates observed were compared to available WHO estimates [28], using the prevalence ratio. Total and subset-specific LTBI prevalence was calculated using as a sample size those who underwent TST without a diagnosis of TB. The number of individuals with LTBI was estimated using the proportion of IGRA-positive individuals out of those TST-positive individuals who were examined at Villa Marelli Institute. This was adjusted by the percentage of those lost to follow-up after a positive TST result, with the assumption that they had the same IGRA-positive proportion. Data were then compared with prevalence rates derived from the literature [29] using a Chi-squared test.

The surveillance system was assessed using the following indicators: completeness of medical evaluation after a positive TST and/or QS result, timing of interventions, TB treatment outcomes, proportion of acceptance and adherence to LTBI preventive therapy, database validity (duplicate records and errors) and internal completeness. A Chi-squared test was used to compare 1) anti-TB treatment success rates based on the country of origin and 2) adverse side effects between those lost to follow-up and those who completed treatment.

\section{Results}

\section{Validity and internal completeness monitoring}

Records included in the database, before data cleaning and record linkage procedures, totalled 7325 . Of them, 1975 (27.0\%) were duplicates while 26 were reception centre staff members screened with TST, and therefore excluded from study analysis. Errors in data collection were detected in $2.2 \%$ of the fields analysed, mainly affecting information in the fields of country of origin $(223 ; 31.9 \%)$ and TST result $(227 ; 32.4 \%)$. 
After comparing the first- and second-level screening system database, a total of 868 (2.7\%) fields were found to be unfilled for the QS score $(517 ; 59.6 \%)$ variable.

\section{First-level surveillance and screening}

During the 2016-2017 study period a total of 5324 asylum seekers were enrolled. 53.4\% were aged 2029 years; most were male (84.2\%) and from sub-Saharan African countries (69.1\%) (table 1). The most frequent countries of origin were Eritrea $(583 ; 11.0 \%)$, Nigeria $(564 ; 10.6 \%)$, Somalia $(474 ; 8.9 \%)$, Pakistan (385; 7.2\%) and Bangladesh (385; 7.2\%). Among the asylum seekers screened with the TST and QS by the health authorities of the city of Milan, 2403 (45.1\%) had positive results (figure 1): 2298 (43.2\%) for the TST and 270 (5.1\%) for the QS, including 165 who had positive results for both tests.

In terms of the main region of origin, positive TST results were found for 1170 asylum seekers (48.5\%) from West Africa (Benin, Burkina Faso, Côte d'Ivoire, Gambia, Ghana, Guinea, Guinea-Bissau, Liberia, Mali, Mauritania, Niger, Nigeria, Senegal, Sierra Leone and Togo), 467 (40.4\%) from East Africa (Eritrea, Ethiopia, Kenya, Somalia, South Sudan, Tanzania and Uganda) and 407 (41.7\%) from South Asia (Afghanistan, Bangladesh, India, Nepal, Pakistan and Sri Lanka). The QS was positive mainly in individuals from North Africa (Egypt, Libya, Morocco, Sudan and Tunisia) (five; 7.7\%) and West Africa $(144 ; 6.0 \%)$.

\section{Second-level surveillance and screening}

During the study period, 69 active TB cases were diagnosed among asylum seekers $(1.3 \%$ of the total asylum seekers screened). Of those, $89.9 \%$ were among individuals aged $10-29$ years, $88.4 \%$ were male and the median time of permanence in Italy was 6 months (range 0.5-36 months). Cases came primarily from West (47.8\%) and East (44.9\%) African countries, mainly Somalia (20;29.0\%), Eritrea (11; 15.9\%), Senegal (seven; $10.1 \%$ ) and Guinea (seven; 10.1\%).

Of the 69 TB cases, 58 (84.1\%) were pulmonary (including those with either pulmonary disease only or both pulmonary and extrapulmonary disease) and 11 (15.9\%) were exclusively extrapulmonary. Of these,

TABLE 1 Baseline characteristics of asylum seeker population within reception centres in Milan participating in screening activities

\begin{tabular}{|c|c|c|c|c|}
\hline & Total & TST-positive ${ }^{\#}$ & QS-positive ${ }^{\#}$ & IGRA-positive/IGRA tested \\
\hline Total & 5324 (100.0) & $2298(43.2)$ & $270(5.1)$ & $865 / 1339(64.6)$ \\
\hline \multicolumn{5}{|l|}{ Sex } \\
\hline Male & 4482 (84.2) & $2003(44.7)$ & 236 (5.3) & $760 / 1189$ (63.9) \\
\hline Female & $734(13.8)$ & $260(35.4)$ & $28(3.8)$ & $105 / 150(70.0)$ \\
\hline Unknown & $108(2.0)$ & $35(32.4)$ & $6(5.6)$ & - \\
\hline \multicolumn{5}{|l|}{ Age years } \\
\hline $0-9$ & $60(1.1)$ & $12(20.0)$ & $1(1.7)$ & $2 / 5(40.0)$ \\
\hline $10-19$ & 1375 (25.8) & $580(42.2)$ & $67(4.9)$ & $232 / 367(63.2)$ \\
\hline $20-29$ & $2843(53.4)$ & 1215 (42.7) & $153(5.4)$ & $494 / 766(64.5)$ \\
\hline$\geqslant 30$ & 985 (18.5) & $464(47.1)$ & $35(3.6)$ & $137 / 201(68.2)$ \\
\hline Unknown & $61(1.1)$ & $27(44.3)$ & $27(44.3)$ & - \\
\hline \multicolumn{5}{|c|}{ Geographical region of origin } \\
\hline North Africa & $299(5.6)$ & 65 (21.7) & $5(7.7)$ & 14/24 (58.3) \\
\hline East Africa & 1155 (21.7) & $467(40.4)$ & $43(3.7)$ & $180 / 221(81.4)$ \\
\hline West Africa & $2415(45.4)$ & $1170(48.5)$ & $144(6.0)$ & 493/757 (65.1) \\
\hline South Asia & $975(18.3)$ & $407(41.7)$ & $42(4.3)$ & $132 / 260(50.8)$ \\
\hline Other & $350(6.6)$ & $124(35.4)$ & $12(3.4)$ & 46/77 (59.7) \\
\hline Unknown & $130(2.4)$ & $65(50.0)$ & $24(18.5)$ & - \\
\hline \multicolumn{5}{|c|}{ WHO region of origin } \\
\hline WHO AFR & 3741 (70.3) & $1711(45.7)$ & $188(5.0)$ & $711 / 1022(69.6)$ \\
\hline WHO EMR & $1041(19.6)$ & $309(29.7)$ & $39(3.8)$ & $98 / 175(56.0)$ \\
\hline Other & $542(10.2)$ & 213 (39.3) & 19 (3.5) & $56 / 142(39.4)$ \\
\hline Unknown & $130(2.4)$ & $65(50.0)$ & 24 (18.5) & - \\
\hline
\end{tabular}

Data presented as n (\%) by row. Missing data for one or more variable are collected as "unknown", except for the missing result of one screening test (TST or QS) in the presence of a positive result in the remaining test. ": includes data of asylum seekers with positive results in both the TST and QS. TST: tuberculin skin test; QS: questionnaire; IGRA: interferon- $\gamma$ release assay; WHO: World Health Organization; WHO AFR: WHO African Region; WHO EMR: WHO Eastern Mediterranean Region. 


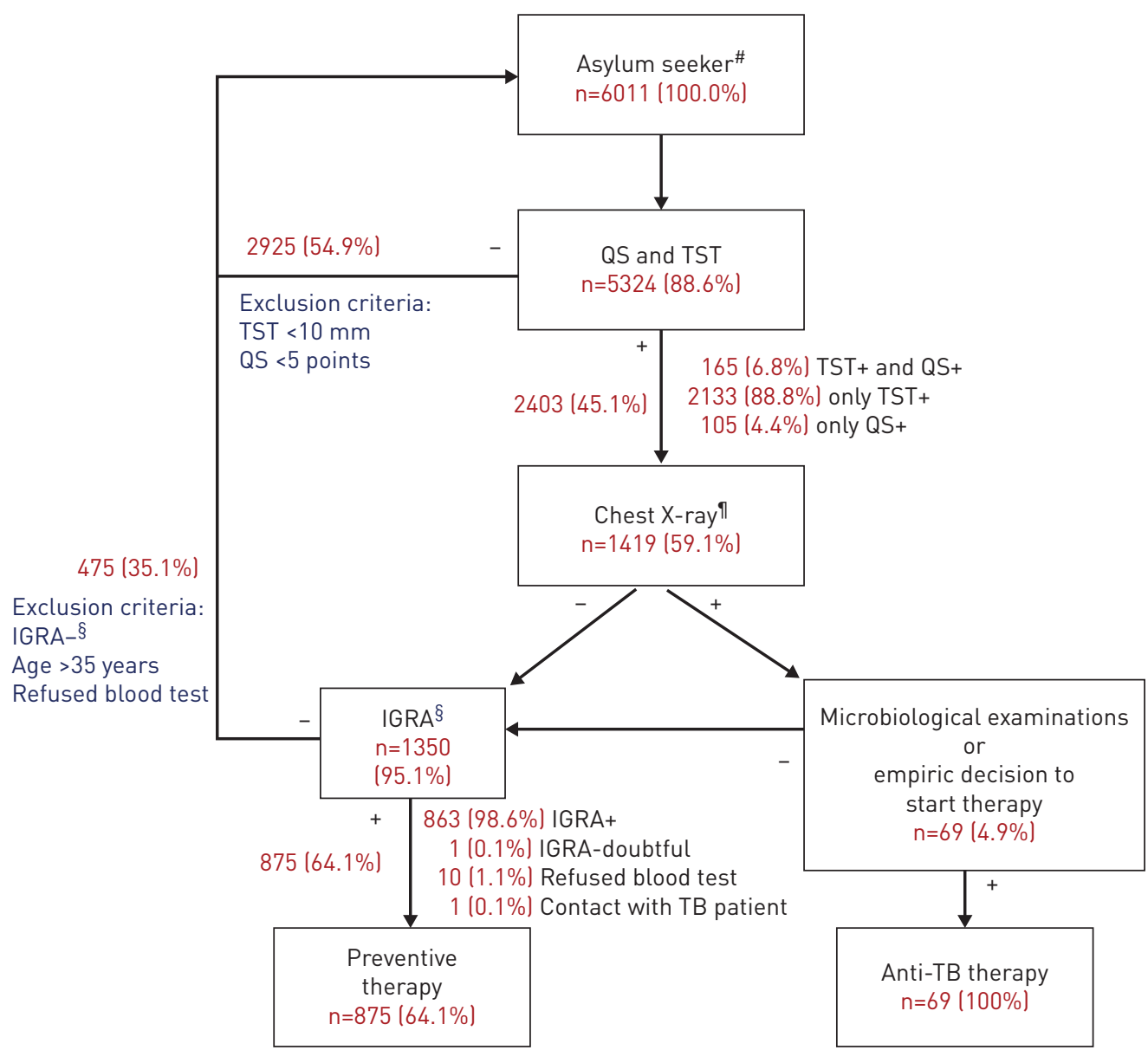

FIGURE 1 Sequence of interventions in screening activities for the diagnosis and treatment of tuberculosis (TB) and latent TB infection (LTBI). Those with negative test results during screening activities include those without data collected in databases. TST: tuberculin skin test; QS: questionnaire. \#: estimated by the municipality of Milan (Comune di Milano); ๆ: data on chest radiography are not routinely collected in the analysed databases, but the number indicated reflects the sum of people tested with interferon- $\gamma$ release assay (IGRA) and those with a diagnosis of TB; ${ }^{\S}$ : IGRA was performed in people aged $<35$ years in the absence of further risk factors (e.g. recent TB contact).

six (54.5\%) had lymph node TB. Among active TB cases, 25 (36.2\%) were classified as possible, with a consequent decision to start therapy, three $(4.4 \%)$ as probable and $41(59.4 \%)$ as culture-confirmed, who then also had a DST.

Drug resistance to at least one first-line anti-TB drug was detected in five cases (12.2\%); two (40.0\%) of them, originating from Liberia and Côte d'Ivoire, were affected by multidrug-resistant TB (MDR-TB). TB/ HIV co-infection was observed in two asylum seekers $(2.9 \%)$.

A total of 1350 individuals were offered IGRA testing. Among them, 10 (0.7\%) refused the IGRA and one child aged 5 years was exempted. Of the 1339 asylum seekers tested, 865 (64.6\%) had a positive IGRA result (274 QFT-GIT and 591 QFT-Plus) (table 1). Among IGRA-positive individuals, two reported a clinical history of previously treated TB; therefore, no preventive treatment was offered.

\section{TB clustering}

TB clustering, proven through the 24-MIRU/VNTR typing system (figure 2), was observed in only two clusters, including five subjects all from East Africa. Cluster 1 included two Somalis hosted in the same reception centre, while Cluster 2 included two Eritreans (A and B) and one Somali (C) hosted by two different reception centres. Despite accurate investigations, no previous or current contact could be demonstrated within Cluster 2 between case $\mathrm{A}$ and the other two (B and $\mathrm{C}$ ). While case $\mathrm{C}$ was assessed to be a recent contact of a non-clustered case (TB_NotClustered, figure 2) by the Health Protection Agency, data provided by molecular testing failed to prove any linkage. 


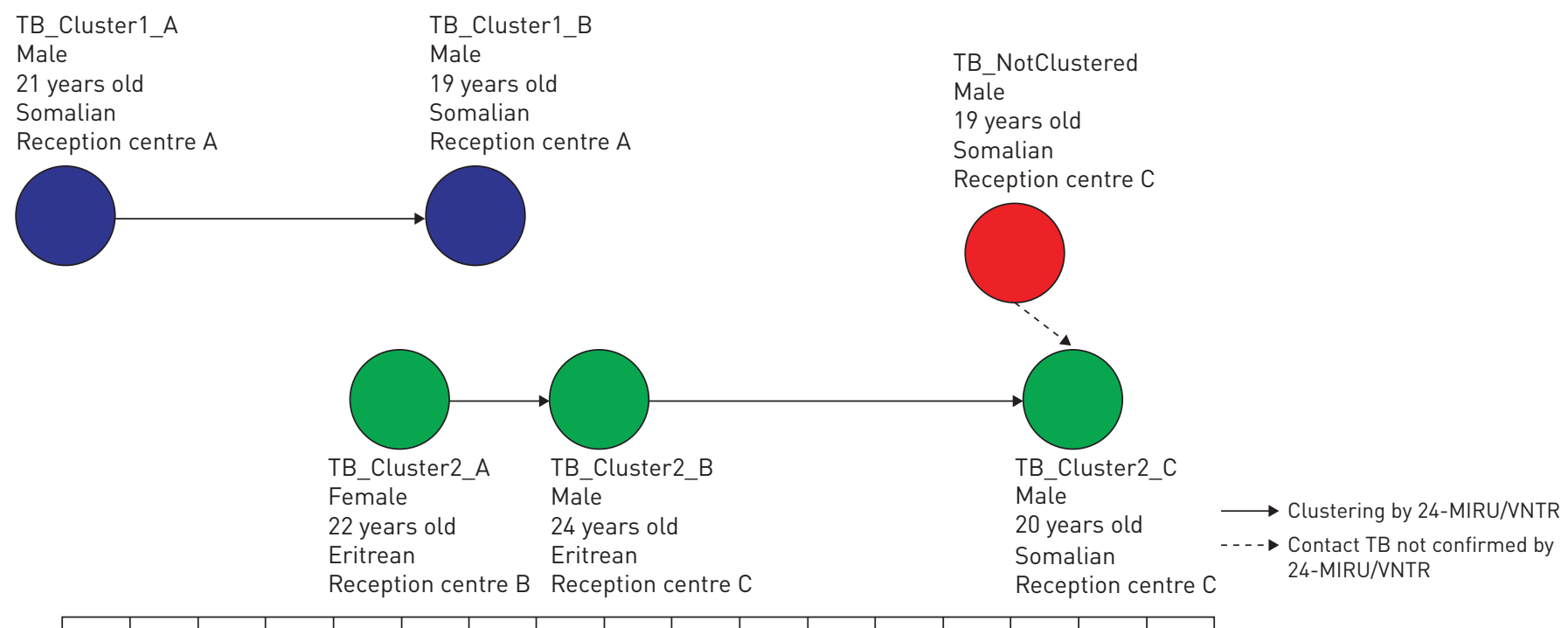

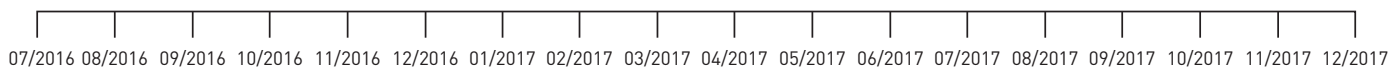

FIGURE 2 Tuberculosis (TB) transmission dynamic within Milan reception centres. Using the 24-locus mycobacterial interspersed repetitive units/ variable-number of tandem repeats (24-MIRU/VNTR) typing system we were able to find five TB cases linked by transmission. However, one

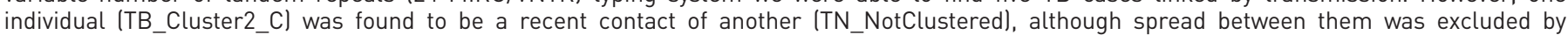
molecular epidemiology.

\section{Active TB prevalence}

The observed overall TB prevalence (table 2) was 1236 per 100000 population (95\% CI 1210-1263 per 100000 population) with differences between WHO regions. Prevalence was 1033 per 100000 population (95\% CI 1006-1060 per 100000 population) among asylum seekers from the WHO African Region (WHO AFR) countries and 3043 per 100000 population (95\% CI 2890-3197 per 100000 population) among those from the WHO Eastern Mediterranean Region (WHO EMR) countries that include Somalia (20 TB cases). The comparison between observed and estimated prevalence showed a higher TB frequency in asylum seekers compared to the general population in the WHO region of origin: the prevalence ratio was 3.1 (95\% CI 2.8-3.5, p<0.001) for WHO AFR and 19.0 (95\% CI 16.2-22.3, p<0.001) for WHO EMR. In particular, the prevalence of TB among asylum seekers from Somalia and Eritrea, jointly responsible for $44.9 \%$ of TB cases, was significantly higher compared with existing estimates, at 3800 per 100000 population (95\% CI 3761-3839 per 100000 population, $\mathrm{p}<0.001$ ) for Somalia and 1658 per 100000 population (95\% CI 1594-1723 per 100000 population, $\mathrm{p}<0.001$ ) for Eritrea [28]. Examining prevalence by geographical region of origin, there were 333 TB cases per 100000 population (95\% CI 315-352 per 100000 population) from Asia, 1379 per 100000 population (95\% CI 1343-2971 per 100000 population) for West Africa and 2971 per 100000 population (95\% CI 2863-3079 per 100000 population) for East Africa.

TABLE 2 TB prevalence among asylum seekers grouped by WHO and geographical regions

Total TB cases $\mathrm{n}(\%)$

$69(100.0)$

33 (47.8)

$31(44.9)$

4 (5.8)

$1(1.4)$

44 (63.8)

$24(34.8)$

$1(1.4)$
TB prevalence per 100000 (95\% CI)

1236 (1210-1263)

$1379(1343-2971)$

2971 (2863-3079)

333 (315-352)

Others

WHO region of origin

WHO AFR

WHO EMR

Others

TB: tuberculosis; WHO: World Health Organization; WHO AFR: WHO African Region; WHO EMR: WHO Eastern Mediterranean Region. 


\section{LTBI prevalence}

Considering only individuals who had positive results for both the IGRA and TST $(844 ; 64.8 \%)$, the LTBI prevalence (table 3) was $27.9 \%$ (95\% CI 26.7\%-29.1\%), differently distributed through regions. The proportion of LTBI in asylum seekers from West Africa $(747 ; 31.4 \%)$ was similar to that in those from East Africa $(372 ; 33.1 \%)$, while a lower proportion was observed in those from Asia $(225 ; 18.6 \%)$. The countries with the highest observed LTBI prevalence were Somalia (193; 42.5\%), Guinea (123; 37.6\%), Nigeria (146; 26.0\%), Eritrea (144; 25.2\%) and Pakistan (94; 24.5\%).

Grouping data using WHO regions, LTBI had a prevalence of $17.4 \%$ (95\% CI 15.1\%-19.8\%) among asylum seekers from WHO EMR, consistent with the $16.3 \%$ (95\% CI 13.4\%-20.5\%, p=0.545) estimated for their countries of origin [29]. A higher prevalence rate $(31.7 \%, 95 \%$ CI $30.2 \%-33.2 \%)$ than the official country estimate $(22.4 \%, 95 \%$ CI $20.6 \%-24.6 \%$, p $<0.001)$ was observed among asylum seekers from WHO AFR [29].

\section{Surveillance and screening participation}

1419 of 2403 asylum seekers (59.1\%) with positive results in screening activities were recorded in the second-level databases, while 984 subjects $(40.9 \%)$ were lost in the transition between the first- and second-level screening.

The median delay between screening activities and second-level tests was 107 days (IQR 47-153 days). It took 28 days (IQR 19-80 days) to receive a result and start treatment after the IGRA. In those who received preventive treatment, the average time between screening and completion was 228 days (158-280 days).

\section{Active TB treatment outcomes}

All 69 patients with an active TB diagnosis were placed on treatment and outcomes were available for 68 (table 4); one MDR-TB patient with HIV co-infection is still receiving treatment. There was a successful outcome in 57 patients $(83.8 \%)$ : in 11 (19.3\%), microbiological confirmation at the end of

TABLE 3 Positivity to TST and IGRA test and LTBI estimation

\begin{tabular}{|c|c|c|c|c|c|c|}
\hline & $\begin{array}{c}\text { A } \\
\text { Total }^{\#} \\
\mathbf{N}\end{array}$ & $\begin{array}{c}\text { B } \\
\text { TST-positive } \\
\text { n (\% A) }\end{array}$ & $\begin{array}{c}\text { C } \\
\text { TST-positive at } \\
\text { second-level screening } \\
n(\% \mathrm{~B})\end{array}$ & $\begin{array}{c}\text { D } \\
\text { IGRA-positive } \\
\text { n }(\% \mathrm{C})\end{array}$ & $\begin{array}{c}\text { E } \\
\text { Estimated } \\
\text { LTBI cases }^{+} \\
\text {n }(\% \text { A })\end{array}$ & $\begin{array}{c}\text { F } \\
\text { Literature estimates } \\
\text { for LTBI prevalence } \\
\%\end{array}$ \\
\hline Total & 5255 & $2259(43.0)$ & $1302(57.6)$ & $844(64.8)$ & $1464(27.9)$ & - \\
\hline \multicolumn{7}{|l|}{$\begin{array}{l}\text { Geographical region and } \\
\text { country of origin }\end{array}$} \\
\hline Nigeria & 562 & $217(38.6)$ & $149(68.7)$ & $100(67.1)$ & $146(26.0)$ & 18 \\
\hline East Africa & 1124 & 448 (39.9) & $212(47.3)$ & $176(83.0)$ & $372(33.1)$ & 22 \\
\hline Eritrea & 572 & $182(31.8)$ & $62(34.1)$ & $49(79.0)$ & $144(25.2)$ & 7 \\
\hline Somalia & 454 & $221(48.7)$ & $126(57.0)$ & $110(87.3)$ & $193(42.5)$ & 22 \\
\hline Asia & 1213 & 465 (38.3) & $291(62.6)$ & $141(48.5)$ & $225(18.6)$ & 27 \\
\hline Bangladesh & 384 & $192(50.0)$ & $124(64.6)$ & 48 (38.7) & 74 (19.3) & 28 \\
\hline WHO AFR & 3697 & $1688(45.7)$ & $997(59.1)$ & 693 (69.5) & $1173(31.7)$ & 22 \\
\hline WHO EMR & 987 & $294(29.8)$ & $166(56.5)$ & 97 (58.4) & $172(17.4)$ & 16 \\
\hline Other & 441 & $217(49.2)$ & $139(64.1)$ & $54(38.8)$ & 84 (19.0) & - \\
\hline Unknown & 130 & 68 (52.3) & - & - & - & - \\
\hline
\end{tabular}

Missing data for one or more variables are collected as "unknown", except for missing result for TST. TST: tuberculin skin test; IGRA: interferon- $\gamma$ release assay; LTBI: latent tuberculosis infection; WHO: World Health Organization; WHO AFR: WHO African Region; WHO EMR: WHO Eastern Mediterranean Region. \#: the total number of individuals (A) was calculated as the total number of subjects who underwent TST minus those with active TB; ${ }^{\uparrow}$ : second-level screening included both chest radiography and IGRA testing, performed at the Villa Marelli Institute; ${ }^{+}$: the estimated prevalence of LTBI (E) was calculated using the \% of IGRA-positive people among the TST-positive people who underwent further testing with IGRA at the Villa Marelli Institute (D/C) applied to the number of those lost between TST and IGRA evaluations $(\times(B-C))$, and assuming they had the same level of positivity as those tested with IGRA. This figure was then added to those with a positive IGRA test (D) to derive the numerator; ${ }^{\S}$ : literature estimates are taken from HouBEN AND DodD [29]. 


\begin{tabular}{lccc}
\multicolumn{2}{c}{ TABLE 4 Treatment outcomes and regimens used for active TB cases } & \\
& Treatment success & Lost to follow-up & Transfer out \\
\hline Anti-TB regimens & $\mathbf{5 7}(\mathbf{8 3 . 8 )}$ & $\mathbf{7}(\mathbf{1 0 . 3 )}$ & $\mathbf{4}$ (5.9) \\
Standard anti-TB regimens & $38(66.7)$ & - & - \\
Modified anti-TB regimens for: & & - & - \\
Adverse drug reaction & $3(5.3)$ & - & - \\
Particular forms of EPTB & $12(21.1)$ & - & - \\
RR/MDR-TB & $2(3.5)$ & - & - \\
No reason reported & $2(3.5)$ & & -
\end{tabular}

Data are presented as $n(\%)$ for $n=68$ cases. TB: tuberculosis; EPTB: extrapulmonary tuberculosis; RR: rifampicin-resistant; MDR: multidrug resistant. " : refers to those with at least 6 months of rifampicin-containing treatment; ": a modified and extended regimen was used for those having particular forms of EPTB (i.e. skeletal, neurological TB).

therapy was obtained, while the remaining 46 (80.7\%) completed the treatment regimen without final microbiological confirmation, mainly due to the lack of capacity to produce sputum. Seven patients (10.3\%) were lost to follow-up, mostly from East African countries (four; 57.1\%). Four patients (5.9\%) were transferred to other treatment sites outside of the study area. No statistical difference was found between treatment success and country of origin for individuals from West $(27 ; 81.8 \%)$ and East $(23$; 74.2\%) Africa ( $\mathrm{p}=0.461)$.

In those completing treatment, 38 (66.7\%) received standard anti-TB regimens of 6-9 months of therapy with an introductory phase with RHZE followed by $\mathrm{RH}(\mathrm{E})$, while $12(21.1 \%)$ with extrapulmonary localisation of the disease (i.e. disseminated and skeletal) received similar treatment but for longer periods. In three individuals (5.3\%) the regimens were modified owing to adverse side effects and in two (3.5\%) owing to drug resistance (rifampicin-resistant/MDR-TB). Two patients (3.5\%) received modified regimens in hospitals different from the Regional TB Reference Centre, and we could not trace the clinical reason underlying these therapeutic choices because they were not reported in the database of the Lombardy region.

\section{LTBI outcomes}

Overall, 875 patients (64.1\%) were eligible for LTBI treatment. The 3-month regimen of rifampicin and isoniazid was used in 863 patients (98.6\%), while $12(1.4 \%)$ were treated with either 4 months of rifampicin or 6 months of isoniazid. The acceptance rate of LTBI treatment was $92.4 \%$, with $4.3 \%$ lost to follow-up after IGRA testing and 3.3\% refusing preventive therapy. Adverse drug reactions developed in 52 individuals (1.9\%) who started treatment, of which 40 cases $(5.2 \%)$ were among the 768 who completed treatment and $12(30.0 \%)$ among the 40 who discontinued treatment $(\mathrm{p}<0.001)$. The final treatment completion rate was $94.3 \%$.

\section{Discussion}

Migrants, particularly asylum seekers, are at great risk of physical and social distress and need to cope with different habits and legislations in new social and cultural situations. In addition, along the migratory route, their health is jeopardised by exposure to risk factors, including violence, malnutrition and environmental challenges [30]. TB is a relevant problem among international migrants [31], and this was confirmed by our study. For these reasons, TB national guidelines [18, 32], adopted in the metropolitan area of Milan, have been implemented to screen migrants for TB and LTBI and, as necessary, treat them.

In the absence of precise information, the number of $\mathrm{TB}$ cases diagnosed among asylum seekers was considered an expression of prevalence, rather than incidence, although most of the individuals had arrived in Italy within 6 months of disease detection. However, it could also be speculated that some of the active cases emerged after arrival in Italy and therefore could represent new incident cases. The impossibility of precisely assigning a date for the onset of symptoms suggests that it is more prudent to consider detected cases as prevalent ones. Therefore, TB prevalence in asylum seekers was found to be remarkably higher than the official WHO estimates [28], suggesting that in some countries the available estimates might be lower than the true TB burden $[13,33]$. This may be particularly true for some African countries for which information is very limited (e.g. Somalia) and estimates are made through expert opinion rather than empirical data $[6,13]$. That notwithstanding, migration is likely a major driving factor for LTBI reactivation, a phenomenon that is well known to persist for several years after arrival [34] as a consequence of marginalisation and poverty. In our study, considering the lack of evidence of recent transmission while in reception sites, the majority of TB cases detected among asylum seekers were 
conceivably due to a state of infection acquired either in the country of origin or along the journey route. This is also supported by the fact that LTBI prevalence among asylum seekers in Milan was very similar to figures observed in previous studies [29, 35, 36].

The evaluation of the TB interventions undertaken in Milan emphasises important achievements, including the high treatment success rate, with almost no transmission documented. Similarly, the very high treatment completion rate of LTBI preventive therapy has potentially averted approximately 80 future TB cases (considering a 10\% rate of LTBI reactivation life-time risk [37]). Furthermore, our assessment of the proportion of individuals with positive IGRA results among those with positive TST results (column $\mathrm{D}$, table 3) suggests that the current screening policy may need adjusting [13]. Modifications, such as the establishment of a single-step approach (TST not followed by IGRA, if positive) in some migrant populations (e.g. East Africans), could be more appropriate especially if backed by cost-effectiveness analysis. The study also highlighted several key areas for strengthening the current surveillance and screening systems. One of the major problems observed in our study was the loss of candidates for preventive therapy between the first and the second step of the system. This is not unlike what has been reported in other studies [15]. We were able to identify some of the organisational factors that jeopardise system performance. They include 1) inadequate data quality control, digitalisation, recording and sharing; 2) high inflows of asylum seekers in Milan during the study period; 3) insufficient engagement of staff at reception centres; 4) lack of coordination due to screening activities dispersed in multiple locations; 5) logistical problems related to transportation of asylum seekers to the clinical services at Villa Marelli Institute; and 6) insufficient coordination among agencies involved.

Correct data registration and digitalisation are essential for data-sharing among agencies, preferably via a common dataset. Therefore, it is crucial that data quality control is developed to ensure that no overestimation of losses recurs in the future [20]. Undoubtedly, the existing system of combined TB control between the Health Protection Agency and the Clinical Reference Centre at Villa Marelli Institute was overstretched once exposed to a large inflow of migrants without a corresponding increase of resources. This led to problems in the correct registration of asylum seekers and a long time interval between the first- and second-level evaluations [38]. Moreover, reception centre staff played a key role in the system because they were accountable for asylum seeker attendance at Villa Marelli Institute. To prevent any withdrawal or delayed appointment for medical evaluation, operators had been informed about the importance of preventing and detecting TB in vulnerable populations. Regrettably, there were frequent logistical and organisational problems resulting in losses or delayed appointments. Additionally, communication between the reception centres and the Villa Marelli Institute and the Health Protection Agency was weak; appointments, notification of delays and cancellations were not systematically collected in a database, resulting in serious gaps in real-time monitoring of data flows and losses.

Finally, the lack of disease knowledge and other cultural issues, e.g. blood stealing/selling beliefs, mostly among West Africans [38, 39], affected acceptance of further confirmatory testing procedures [38].

Besides the issue of clinical evaluation completion, further problems exist that limit TB and LTBI treatment success. For instance, TB treatment success, although satisfactory, could improve substantially if losses to follow-up and transfer out were reduced. The major structural obstacle to treatment completion found in our study was the short notice of relocation/resettlement procedures of asylum seekers within Italy (75\% of those transferred out) or to other EU Member States [14].

Several interventions could be used to implement community-based approaches and improve structural policy. For instance, automated messages could be sent to clinicians asking them to closely oversee the treatment course and correctly report the outcome [20]. Building migrant-sensitive care services and correcting the lack of cross-agency coordination [38] could allow a shared action plan to be developed, thus minimising attrition.

In low-incidence settings aiming at TB elimination, LTBI treatment is essential. A critical step is ensuring completion of therapy once started, because important losses have been observed in several studies [15]. These studies have reported that only half of migrants and other marginalised groups with a medical indication start treatment, usually with very low completion rates. In this study, despite the initial losses during screening, once treatment started all efforts were focused on completion through a migrant-sensitive approach, focusing on counselling about the importance of treatment, directly dispensing medications and involving the reception centre operators in supporting the intervention [38, 40]. An important factor for treatment adherence is the adoption of the short-course treatment ( 3 months of combined rifampicin and isoniazid $\left(\right.$ Rifinah $\left.\left.^{\star}\right)\right)[15,16,41]$.

Our results prompt consideration of the best methods for approaching hard-to-reach populations in a metropolitan area. These may include the creation of a single-centre multidisciplinary service that combines 
the two steps of the actual screening system, leading to a reduction of dilution of health interventions and providing a better standardised method of collecting, recording and sharing data. A mobile system able to bring healthcare workers together with first- and second-line screening activities such as TST, chest radiography and blood tests (including IGRAs) within reception centres would better fit a vulnerable and mobile population. This option may improve completion of clinical evaluation by substantially reducing waiting time and permitting more effective approaches for hard-to-reach communities $[15,31]$ that are heterogeneously distributed within metropolitan areas. A national web-based database would also contribute to reduce losses and duplications and would provide uninterrupted care in mobile populations.

In conclusion, this study shows that well-coordinated screening measures are critical to early diagnosis and treatment among a high-risk, vulnerable and marginalised migrant population in a large metropolitan area. It also proves that rolling out successful at-scale interventions for both preventive therapy and active disease management, and maximising effectiveness through digital innovations allowing better inter-connectivity among involved services, are critical innovations to pursue.

Acknowledgements: We are grateful to all the public health nurses of the Health Protection Agency and the Villa Marelli Institute, who strenuously guaranteed the functioning of the active surveillance and screening system developed in the Milan area.

Conflict of interest: None declared.

\section{References}

1 Odone A, Tillmann T, Sandgren A, et al. Tuberculosis among migrant populations in the European Union and the European Economic Area. Eur J Public Health 2015; 25: 506-512.

2 van Hest N, Aldridge R, de Vries G, et al. Tuberculosis control in big cities and urban risk groups in the European Union: a consensus statement. Euro Surveill 2014; 19: 20728.

3 De Vries G, Van Hest NAH, Baars HWM, et al. Factors associated with the high tuberculosis case rate in an urban area. Int J Tuberc Lung Dis 2010; 14: 859-865.

4 European Centre for Disease Prevention and Control, World Health Organization Regional Office for Europe. Tuberculosis Surveillance and Monitoring in Europe - 2018 (2016 data). Stockholm/Copenhagen, European Centre for Disease Prevention and Control, 2018.

5 EuroTB and the National Coordinators for Tuberculosis Surveillance in the WHO European Region. Surveillance of tuberculosis in Europe. Report on tuberculosis cases notified in 2006. Saint-Maurice, Institut de Veille Sanitaire, 2008.

6 World Health Organization. Global Tuberculosis Report 2018. Geneva, World Health Organization, 2018.

7 Bozorgmehr K, Joggerst B, Wagner $\mathrm{U}$, et al. Yield of tuberculosis screening in asylum-seekers by country of origin: analysis of screening data in a German federal state (2002-2015). Eur Respir J 2017; 49: 1602327.

8 Dara M, Gushulak BD, Posey DL, et al. The history and evolution of immigration medical screening for tuberculosis. Expert Rev Anti Infect Ther 2013; 11: 137-146.

9 Duarte R, Lönnroth K, Carvalho C, et al. Tuberculosis, social determinants and co-morbidities (including HIV). Pulmonology 2018; 24: 115-119.

10 Lönnroth K, Jaramillo E, Williams BG, et al. Drivers of tuberculosis epidemics: the role of risk factors and social determinants. Soc Sci Med 2009; 68: 2240-2246.

11 World Health Organization Regional Office for Europe. Strategy and action plan for refugee and migrant health in the WHO European Region. Copenhagen, World Health Organization, 2016.

12 Kunst H, Burman M, Arnesen TM, et al. Tuberculosis and latent tuberculous infection screening of migrants in Europe: comparative analysis of policies, surveillance systems and results. Int J Tuberc Lung Dis 2017; 21: 840-851.

13 Barcellini L, Campisi D, Castellotti PF, et al. Latent tuberculous infection among foreign-born individuals applying to shelters in the metropolitan area of Milan. Int J Tuberc Lung Dis 2018; 22: 1160-1165.

14 Kuehne A, Hauer B, Brodhun B, et al. Find and treat or find and lose? Tuberculosis treatment outcomes among screened newly arrived asylum seekers in Germany 2002 to 2014. Euro Surveill 2018; 23: 17-00042.

15 Alsdurf H, Hill PC, Matteelli A, et al. The cascade of care in diagnosis and treatment of latent tuberculosis infection: a systematic review and meta-analysis. Lancet Infect Dis 2016; 16: 1269-1278.

16 Sandgren A, Vonk Noordegraaf-Schouten M, van Kessel F, et al. Initiation and completion rates for latent tuberculosis infection treatment: a systematic review. BMC Infect Dis 2016; 16: 204.

17 Heuvelings CC, de Vries SG, Grobusch MP. Tackling TB in low-incidence countries: improving diagnosis and management in vulnerable populations. Int J Infect Dis 2017; 54: 77-80.

18 Giunta Regionale E Assessori. Revisione ed aggiornamento degli interventi di sorveglianza, prevenzione, profilassi e controllo della tubercolosi in Regione Lombardia [Update on LTBI prevention, TB surveillance and control in the Lombardy region]. Regione Lombardia Bollettino Ufficiale 2017; n. X/6855. www.regione.lombardia.it/wps/ wcm/connect/e8405eb0-2f52-473c-9c9e-2a565a019bae/SEO29_18-07-2017.pdf?MOD=AJPERES\&CACHEID=e840 5eb0-2f52-473c-9c9e-2a565a019bae Date last accessed: June 14, 2019.

19 European Centre for Disease Prevention and Control. Data quality monitoring and surveillance system evaluation a handbook of methods and applications. Stockholm, European Centre for Disease Prevention and Control, 2014.

20 Schneeberger Geisler S, Helbling P, Zellweger JP, et al. Screening for tuberculosis in asylum seekers: comparison of chest radiography with an interview-based system. Int J Tuberc Lung Dis 2010; 14: 1388-1394.

21 European Commission. Commission Implementing Decision (EU) 2018/945 of 22 June 2018 on the communicable diseases and related special health issues to be covered by epidemiological surveillance as well as relevant case definitions. Off J Eur Union 2018; 61: 1-74.

22 Weyer K, Mirzayev F, Migliori GB, et al. Rapid molecular TB diagnosis: evidence, policy making and global implementation of Xpert MTB/RIF. Eur Respir J 2013; 42: 252-271. 
23 World Health Organization. Definitions and reporting framework for tuberculosis - 2013 revision. Geneva, World Health Organization, 2014.

24 World Health Organization. Treatment of tuberculosis: guidelines - 4th ed. Geneva, World Health Organization, 2010.

25 Supply P, Allix C, Lesjean S, et al. Proposal for standardization of optimized mycobacterial interspersed repetitive unit-variable-number tandem repeat typing of Mycobacterium tuberculosis. J Clin Microbiol 2006; 44: 4498-4510.

26 Allix-Béguec C, Supply P, Wanlin M, et al. Standardised PCR-based molecular epidemiology of tuberculosis. Eur Respir J 2008; 31: 1077-1084.

27 Barcellini L, Borroni E, Brown J, et al. First evaluation of QuantiFERON-TB gold plus performance in contact screening. Eur Respir J 2016; 48: 1411-1419.

28 World Health Organization. Global Tuberculosis Report 2015. Geneva, World Health Organization, 2015.

29 Houben RMGJ, Dodd PJ. The global burden of latent tuberculosis infection: a re-estimation using mathematical modelling. PLoS Med 2016; 13: e1002152.

30 Sotgiu G, Dara M, Centis R, et al. Breaking the barriers: migrants and tuberculosis. Press Medicale 2017; 46: e5-e11.

31 Eiset $\mathrm{AH}$, Wejse C. Review of infectious diseases in refugees and asylum seekers-current status and going forward. Public Health Rev 2017; 38: 22.

32 INMP/NIHMP, ISS, SIMM. I controlli alla frontiera - La frontiera dei controlli [The frontier of controls - Controls at the frontier]. 2017. www.salute.gov.it/imgs/C_17_pubblicazioni_2624_allegato.pdf Date last accessed: June 3, 2019.

33 Zumla A, Petersen E, Nyirenda T, et al. Tackling the tuberculosis epidemic in Sub-Saharan Africa - unique opportunities arising from the second European Developing Countries Clinical Trials Partnership (EDCTP) programme 2015-2024. Int J Infect Dis 2015; 32: 46-49.

34 Lillebaek T, Andersen ÅB, Dirksen A, et al. Persistent high incidence of tuberculosis in immigrants in a low-incidence country. Emerging Infect Dis 2002; 8: 679-668.

35 Sarivalasis A, Zellweger J-P, Faouzi M, et al. Factors associated with latent tuberculosis among asylum seekers in Switzerland: a cross-sectional study in Vaud County. BMC Infect Dis 2012; 12: 285.

36 Vanino E, Tadolini M, Attard L, et al. Systematic tuberculosis screening in asylum seekers in Italy. Clin Infect Dis 2017; 65: 1407-1409.

37 Mack U, Migliori GB, Sester M, et al. LTBI: latent tuberculosis infection or lasting immune responses to M. tuberculosis? A TBNET consensus statement. Eur Respir J 2009; 33: 956-973.

38 Dhavan P, Dias HM, Creswell J, et al. An overview of tuberculosis and migration. Int J Tuberc Lung Dis 2017; 21: 610-623.

39 Grietens KP, Ribera JM, Erhart A, et al. Doctors and vampires in sub-Saharan Africa: ethical challenges in clinical trial research. Am J Trop Med Hyg 2014; 91: 213-215.

40 Lutge EE, Wiysonge CS, Knight SE, et al. Material incentives and enablers in the management of tuberculosis. Cochrane Database Syst Rev 2012; 1: CD007952.

41 Pai M, Behr MA, Dowdy D, et al. Tuberculosis review. Nat Rev Dis Prim 2016; 2: 16076. 